\title{
ON CERTAIN LINEAR STRUCTURES OF BOUNDED VECTOR - VALUED SEQUENCE SPACE ON PRODUCT NORMED SPACE
}

\author{
Narayan Prasad Pahari \\ Central Department of Mathematics, Tribhuvan University, Kirtipur, Kathmandu, Nepal \\ Email Address: nppahari@gmail.com
}

\begin{abstract}
The aim of this paper is to dealwith avector valued sequence space $\mathrm{V}(\mathrm{P}, \bar{\gamma}, \overline{\mathrm{u}},\|\cdot\|)$ with its terms from a product normed space P. Beside investigating the linear space structure of $\mathrm{V}(\mathrm{P}, \bar{\gamma}, \overline{\mathrm{u}}, \|$. $\|)$ with respect to co-ordinate-wise vector operations, the primary interest is to explore the conditions in terms of $\overline{\mathrm{u}}$ and $\bar{\gamma}$ so that a class $\mathrm{V}(\mathrm{P}, \bar{\gamma}, \overline{\mathrm{u}},\|\cdot\|)$ is contained in or equal to another class of the same kind .
\end{abstract}

Keywords: Sequence space, Generalized sequence space, Product normed space.

\section{Introduction and Preliminaries}

So far a bulk number of research works have been done on Bounded Vector -Valued Sequence Space on Product Normed Space. The notion of vector valued sequence space is a generalized form of spaces of scalar valued sequences, and its terms consist of sequences from a vector space. The various types of vector valued sequence spaces has been significantly developed by several workers for instances, Köthe (1970), Kamthan and Gupta (1980), Maddox (1980), Ruckle (1981), Malkowski and Rakocevic (2004), Khan (2008), Kolk (2011), Srivastava and Pahari (2012) etc and others.

Let $\mathrm{X}$ be a normed space over $\mid$, the field of complex numbers and let $\omega(X)$ denotes the linear space of all sequences $\overline{\mathrm{x}}=\left(\mathrm{x}_{\mathrm{k}}\right)$ with $\mathrm{x}_{\mathrm{k}} \in \mathrm{X}, \mathrm{k} \geq 1$ with usual coordinate-wise operations .We shall denote $\omega(\mathbf{C})$ by $\omega$. Any subspace $S$ of $\omega$ is then called a sequence space. A vector valued sequence space or a generalized sequence space is a linear space consisting of sequences with their terms from a vector space.

Let $\left(\mathrm{X},\|\cdot\|_{\mathrm{X}}\right)$ and $\left(\mathrm{Y},\|\cdot\|_{\mathrm{Y}}\right)$ be Banach spaces over the field | of complex numbers. Clearly the linear space structure of $\mathrm{X}$ and $\mathrm{Y}$ provides the Cartesian product of $\mathrm{X}$ and $\mathrm{Y}$ given by

$$
\mathrm{X} \times \mathrm{Y}=\{<\mathrm{x}, \mathrm{y}>: \mathrm{x} \in \mathrm{X}, \mathrm{y} \in \mathrm{Y}\}
$$

forms a normed linear space over | under the algebraic operations

$$
\begin{aligned}
<\mathrm{x}_{1}, \mathrm{y}_{1}>+<\mathrm{x}_{2}, \mathrm{y}_{2}>=<\mathrm{x}_{1}+\mathrm{x}_{2}, \mathrm{y}_{1}+\mathrm{y}_{2}> \\
\\
\quad \text { and } \alpha<\mathrm{x}, \mathrm{y}>=<\alpha \mathrm{x}, \alpha \mathrm{y}>
\end{aligned}
$$

with the norm

$$
\|<\mathrm{x}, \mathrm{y}>\|=\max \left\{\|\mathrm{x}\|_{\mathrm{x}},\|\mathrm{y}\|_{\mathrm{Y}}\right\}
$$

where $\left\langle\mathrm{x}_{1}, \mathrm{y}_{1}>,<\mathrm{x}_{2}, \mathrm{y}_{2}>,<\mathrm{x}, \mathrm{y}\right\rangle \in \mathrm{X} \times \mathrm{Y}$ and $\alpha \in \mid$.

Throughout the work, we denote the product space $\mathrm{X} \times \mathrm{Y}$ by $\mathrm{P}$. 
Moreover since $\left(\mathrm{X},\|\cdot\|_{\mathrm{X}}\right)$ and $\left(\mathrm{Y},\|\cdot\|_{\mathrm{Y}}\right)$ are Banach spaces therefore $(\mathrm{X} \times \mathrm{Y},\|<\ldots, .>\|)$ is also a Banach space

Sanchezl et al(2000), Castillo et al (2001) and Yilmaz et al(2004) and many others have introduced and examined some properties of bilinear vector valued sequence spaces defined on product normed space which generalize many sequence spaces.

\section{The Space $\mathbf{V}(\mathbf{P}, \bar{\gamma}, \overline{\mathbf{u}},\|\cdot\|)$ on Product Normed Space}

Let $\overline{\mathrm{u}}=\left(\mathrm{u}_{\mathrm{k}}\right)$ and $\overline{\mathrm{v}}=\left(\mathrm{v}_{\mathrm{k}}\right)$ be any sequences of strictly positive real numbers and $\bar{\gamma}=\left(\gamma_{\mathrm{k}}\right)$ and $\bar{\mu}=\left(\mu_{\mathrm{k}}\right)$ be sequences of non-zero complex numbers.

We now introduce and study the following class of Banach space $\mathrm{X} \times \mathrm{Y}$-valued sequences:

$$
\mathrm{V}(\mathrm{P}, \bar{\gamma}, \overline{\mathrm{u}},\|\cdot\|)=\left\{\overline{\mathrm{u}}=\left(<\mathrm{x}_{\mathrm{k}}, \mathrm{y}_{\mathrm{k}}>\right):<\mathrm{x}_{\mathrm{k}}, \mathrm{y}_{\mathrm{k}}>\in \mathrm{X} \times \mathrm{Y}, \quad \sup _{\mathrm{k}}\left\|\gamma_{\mathrm{k}}<\mathrm{x}_{\mathrm{k}}, \mathrm{y}_{\mathrm{k}}>\right\|^{\mathrm{uk}}<\infty\right\}
$$

Further, when $\gamma_{\mathrm{k}}=1$ for all $\mathrm{k}$, then $\mathrm{V}(\mathrm{P}, \bar{\gamma}, \overline{\mathrm{u}},\|\cdot\|)$ will be denoted by $\mathrm{V}(\mathrm{P}, \overline{\mathrm{u}},\|\cdot\|)$ and when $\mathrm{u}_{\mathrm{k}}=1$ for all $\mathrm{k}$ then $\mathrm{V}(\mathrm{P}, \bar{\gamma}, \overline{\mathrm{u}},\|\cdot\|)$ will be denoted by $\mathrm{V}(\mathrm{P}, \bar{\gamma},\|\cdot\|)$.

In fact, this class is the generalization of the space introduced and studied by Srivastava and Pahari (2012) to the product normed space..

\section{Linear Topological Structures}

In this section we shall derive the linear space structure of the class V (P, $\bar{\gamma}, \overline{\mathrm{u}},\|\cdot\|$ ) over the field $\mathbf{C}$ of complex numbers and thereby investigate conditions in terms of $\bar{u}, \bar{v}, \bar{\gamma}$ and $\bar{\mu}$ so that a class is contained in or equal to another class of same kind .As far as the linear space structure of $\mathrm{V}(\mathrm{P}, \bar{\gamma}, \overline{\mathrm{u}}, \|$. $\|$ ) over $\mathbf{C}$ is concerned we throughout take the co-ordinate wise vector operations i.e., for $\overline{\mathrm{w}}=\left(<\mathrm{x}_{\mathrm{k}}\right.$ , $\left.\mathrm{y}_{\mathrm{k}}>\right), \overline{\mathrm{z}}=\left(<\mathrm{x}_{\mathrm{k}}^{\prime}, \mathrm{y}_{\mathrm{k}}^{\prime}>\right)$ in $\mathrm{V}(\mathrm{P}, \bar{\gamma}, \overline{\mathrm{u}},\|\cdot\|)$ and scalar $\alpha$, we have

$$
\begin{aligned}
& \overline{\mathrm{w}}+\overline{\mathrm{z}}=\left(<\mathrm{x}_{\mathrm{k}}, \mathrm{y}_{\mathrm{k}}>\right)+\left(<\mathrm{x}_{\mathrm{k}}^{\prime}, \mathrm{y}_{\mathrm{k}}^{\prime}>\right)=\left(<\mathrm{x}_{\mathrm{k}}+\mathrm{x}_{\mathrm{k}}^{\prime}, \mathrm{y}_{\mathrm{k}}+\mathrm{y}_{\mathrm{k}}^{\prime}>\right) \\
& \text { and } \\
& \alpha \alpha \overline{\mathrm{u}}=\left(\alpha<\mathrm{x}_{\mathrm{k}}, \mathrm{y}_{\mathrm{k}}>\right)=\left(<\alpha \mathrm{x}_{\mathrm{k}}, \alpha \mathrm{y}_{\mathrm{k}}>\right) .
\end{aligned}
$$

Further, by $\overline{\mathrm{u}}=\left(\mathrm{u}_{\mathrm{k}}\right) \in \ell_{\infty}$, we mean $\sup _{k} \mathrm{u}_{\mathrm{k}}<\infty$ and we see below that $\sup _{k} \mathrm{u}_{\mathrm{k}}<\infty$ is the necessary condition for linearity of the space.

We shall denote $M=\max \left(1, \sup _{k} u_{k}\right)$ and $A(\alpha)=\max (1,|\alpha|)$. The zero element of the space will be denoted by

$\bar{\theta}=(<0,0>,<0,0>,<0,0>, \ldots .)$. 
Theorem 3.1: If $\overline{\mathbf{u}}=\left(\mathbf{u}_{\mathrm{k}}\right) \in \ell_{\infty}$, then $\mathrm{V}(\mathbf{P}, \bar{\gamma}, \overline{\mathbf{u}},\|\cdot\|)$ forms a vector space over $\mathrm{C}$.

Proof:

Assume that $\overline{\mathrm{u}}=\left(\mathrm{u}_{\mathrm{k}}\right) \in \ell_{\infty}$ and $\overline{\mathrm{w}}=\left(<\mathrm{x}_{\mathrm{k}}, \mathrm{y}_{\mathrm{k}}>\right)$ and $\overline{\mathrm{z}}=\left(<\mathrm{x}_{\mathrm{k}}^{\prime}, \mathrm{y}_{\mathrm{k}}^{\prime}>\right) \in \mathrm{V}(\mathrm{P}, \bar{\gamma}, \overline{\mathrm{u}},\|\cdot\|)$.So that we have

$\sup _{k}\left\|\gamma_{\mathrm{k}}<\mathrm{x}_{\mathrm{k}}, \mathrm{y}_{\mathrm{k}}>\right\|^{\mathrm{uk}}<\infty$ and $\sup _{k}\left\|\gamma_{\mathrm{k}}<\mathrm{x}_{\mathrm{k}}^{\prime}, \mathrm{y}_{\mathrm{k}}^{\prime}>\right\|^{\mathrm{uk}}<\infty$.

Thus considering

$$
\sup _{k}\left\|\gamma_{\mathrm{k}}\left(<\mathrm{x}_{\mathrm{k}}, \mathrm{y}_{\mathrm{k}}>+<\mathrm{x}_{\mathrm{k}}^{\prime}, \mathrm{y}_{\mathrm{k}}^{\prime}>\right)\right\|^{\mathrm{uk} / \mathrm{M}} \leq \sup _{k}\left\|\gamma_{\mathrm{k}}<\mathrm{x}_{\mathrm{k}}, \mathrm{y}_{\mathrm{k}}>\right\|^{\mathrm{uk} / \mathrm{M}}+\sup _{k}\left\|\gamma_{\mathrm{k}}<\mathrm{x}_{\mathrm{k}}^{\prime}, \mathrm{y}_{\mathrm{k}}^{\prime}>\right\|^{\mathrm{uk} / \mathrm{M}}
$$

and we see that $\sup _{k}\left\|\gamma_{k}\left(<x_{k}, y_{k}>+<x_{k}^{\prime}, y_{k}^{\prime}>\right)\right\|^{\mathrm{uk} / M}<\infty$

and hence $\overline{\mathrm{w}}+\overline{\mathrm{z}} \in \mathrm{V}(\mathrm{P}, \bar{\gamma}, \overline{\mathrm{u}},\|\cdot\|)$. Similarly for any scalar $\alpha, \alpha \overline{\mathrm{w}} \in \mathrm{V}(\mathrm{P}, \bar{\gamma}, \overline{\mathrm{u}},\|\cdot\|)$ since

$$
\begin{aligned}
& \sup _{k}\left\|\alpha \gamma_{\mathrm{k}}<\mathrm{x}_{\mathrm{k}}, \mathrm{y}_{\mathrm{k}}>\right\|^{\mathrm{uk} / \mathrm{M}}=\sup _{k}|\alpha|^{\mathrm{uk} / \mathrm{M}}\left\|\gamma_{\mathrm{k}}<\mathrm{x}_{\mathrm{k}}, \mathrm{y}_{\mathrm{k}}>\right\|^{\mathrm{uk} / \mathrm{M}} \\
& \leq \mathrm{A}(\alpha) \sup _{k}\left\|\gamma_{\mathrm{k}}<\mathrm{x}_{\mathrm{k}}, \mathrm{y}_{\mathrm{k}}>\right\|^{\mathrm{uk} / \mathrm{M}}<\infty .
\end{aligned}
$$

Theorem 3.2: $\mathbf{V}(\mathbf{P}, \bar{\gamma}, \overline{\mathbf{u}},\|\cdot\|)$ forms a vector space over $\mathbf{C}$ if $\overline{\mathbf{u}}=\left(\mathbf{u}_{\mathrm{k}}\right) \in \ell_{\infty}$.

\section{Proof:}

Suppose $\overline{\mathrm{u}}=\left(\mathrm{u}_{\mathrm{k}}\right) \notin \ell_{\infty}$ then we can find a sequence $(\mathrm{k}(\mathrm{n}))$ of positive integers with

$$
\mathrm{k}(\mathrm{n})<\mathrm{k}(\mathrm{n}+1), \mathrm{n} \geq 1
$$

such that $\mathrm{u}_{\mathrm{k}(\mathrm{n})}>\mathrm{n}$ for each $\mathrm{n} \geq 1$. Now taking $<\mathrm{r}, \mathrm{t}>\in \mathrm{X} \times \mathrm{Y},\|<\mathrm{r}, \mathrm{t}>\|=1$ we define a sequence $\overline{\mathrm{w}}=\left(<\mathrm{x}_{\mathrm{k}}, \mathrm{y}_{\mathrm{k}}>\right)$ by

$$
\begin{aligned}
& <\mathrm{x}_{\mathrm{k}}, \mathrm{y}_{\mathrm{k}}>=\left\{\begin{array}{l}
\lambda_{\mathrm{k}(\mathrm{n})}^{-1} \mathrm{n}^{-\mathrm{rk}(\mathrm{n})}<\mathrm{r}, \mathrm{t}>, \text { for } \mathrm{k}=\mathrm{k}(\mathrm{n}), \mathrm{n} \geq 1 \text {, and } \\
<0,0>\text {, otherwise. }
\end{array}\right. \\
& \text { where }<\mathrm{r}, \mathrm{t}>\in \mathrm{X} \times \mathrm{Y} \text { with }\|<\mathrm{r}, \mathrm{t}>\|=1 \text {, then we have } \\
& \sup _{k}\left\|\gamma_{\mathrm{k}}<\mathrm{x}_{\mathrm{k}}, \mathrm{y}_{\mathrm{k}}>\right\|^{\mathrm{uk}}=\sup _{n}\left\|\gamma_{\mathrm{k}(\mathrm{n})}<\mathrm{x}_{\mathrm{k}(\mathrm{n})}, \mathrm{y}_{\mathrm{k}(\mathrm{n})}>\right\|^{\mathrm{uk}(\mathrm{n})} \\
& =\sup _{n}\left\|\mathrm{n}^{-\mathrm{rk}(\mathrm{n})}<\mathrm{r}, \mathrm{t}>\right\|^{\mathrm{uk}(\mathrm{n})} \\
& =\sup _{n} \frac{1}{n}=1 \text {. }
\end{aligned}
$$

Thus we easily see that $\overline{\mathrm{w}} \in \mathrm{V}(\mathrm{X} \times \mathrm{Y}, \bar{\gamma}, \overline{\mathrm{u}},\|\cdot\|)$ but on the other hand for $\mathrm{k}=\mathrm{k}(\mathrm{n}), \mathrm{n} \geq 1$ and for the scalar

$\alpha=2$,we have

$$
\sup _{k}\left\|\gamma_{\mathrm{k}}\left(\alpha<\mathrm{x}_{\mathrm{k}}, \mathrm{y}_{\mathrm{k}}>\right)\right\|^{\mathrm{uk}}=\sup _{k}\left\|\gamma_{\mathrm{k}(\mathrm{n})}\left(\alpha<\mathrm{x}_{\mathrm{k}(\mathrm{n})}, \mathrm{y}_{\mathrm{k}(\mathrm{n})}>\right)\right\|^{\mathrm{uk}(\mathrm{n})}
$$




$$
\begin{gathered}
=\sup _{n}|2|^{\mathrm{uk(n)}}\left\|\mathrm{n}^{-\mathrm{rk}(\mathrm{n})}<\mathrm{r}, \mathrm{t}>\right\|^{\mathrm{uk}(\mathrm{n})} \\
=\sup _{n}|2|^{\mathrm{uk(n)}} \cdot \frac{1}{\mathrm{n}}>\sup _{n} \frac{2^{\mathrm{n}}}{\mathrm{n}} \geq 1
\end{gathered}
$$

This shows that $\alpha \overline{\mathrm{w}} \notin \mathrm{V}(\mathrm{P}, \bar{\gamma}, \overline{\mathrm{u}},\|\cdot\|)$. Hence $\mathrm{V}(\mathrm{P}, \bar{\gamma}, \overline{\mathrm{u}},\|\cdot\|)$ will form linear space if and only if $\overline{\mathrm{u}}=\left(\mathrm{u}_{\mathrm{k}}\right) \in \ell_{\infty}$.

Theorem 3.3: For any $\overline{\mathrm{u}}=\left(\mathrm{u}_{\mathrm{k}}\right), \mathrm{V}(\mathrm{P}, \bar{\gamma}, \overline{\mathrm{u}},\|\cdot\|) \subset \mathrm{V}(\mathrm{P}, \bar{\mu}, \overline{\mathrm{u}},\|\cdot\|)$ if and only if $\lim \inf _{k}\left|\frac{\gamma_{\mathrm{k}}}{\mu_{\mathrm{k}}}\right|^{\mathrm{uk}}>0$

\section{Proof :}

Suppose $\lim \inf _{k}\left|\frac{\gamma_{k}}{\mu_{k}}\right|^{\text {uk }}>0$, and $\bar{w}=\left(<x_{k}, y_{k}>\right) \in V(P, \bar{\gamma}, \bar{u},\|\cdot\|)$. Then there exists $m>0$, such that

$\mathrm{m}\left|\mu_{\mathrm{k}}\right|^{\mathrm{uk}}<\left|\gamma_{\mathrm{k}}\right|^{\mathrm{uk}}$

for all sufficiently large values of $k$. Thus

$$
\sup _{k}\left\|\mu_{\mathrm{k}}<\mathrm{x}_{\mathrm{k}}, \mathrm{y}_{\mathrm{k}}>\right\|^{\mathrm{uk}} \leq \sup _{k} \frac{1}{\mathrm{~m}}\left\|\gamma_{\mathrm{k}}<\mathrm{x}_{\mathrm{k}}, \mathrm{y}_{\mathrm{k}}>\right\|^{\mathrm{uk}}<\infty
$$

for all sufficiently large values of $k$, implies that $\overline{\mathrm{w}} \in \mathrm{V}(\mathrm{P}, \bar{\mu}, \overline{\mathrm{u}},\|\cdot\|)$. Hence

$\mathrm{V}(\mathrm{P}, \bar{\gamma}, \overline{\mathrm{u}},\|\cdot\|) \subset \mathrm{V}(\mathrm{P}, \bar{\mu}, \overline{\mathrm{u}},\|\cdot\|)$.

Conversely, let

$\mathrm{V}(\mathrm{P}, \bar{\gamma}, \overline{\mathrm{u}},\|\cdot\|) \subset \mathrm{V}(\mathrm{P}, \bar{\mu}, \overline{\mathrm{u}},\|\cdot\|)$

But $\lim \inf _{k}\left|\frac{\gamma_{k}}{\mu_{k}}\right|^{\text {uk }}=0$. Then we can find a sequence $(k(n))$ of positive integers with

$\mathrm{k}(\mathrm{n})<\mathrm{k}(\mathrm{n}+1), \mathrm{n} \geq 1$

such that

$$
\left|\mu_{\mathrm{k}(\mathrm{n})}\right|^{\mathrm{uk}(\mathrm{n})}>\mathrm{n}\left|\gamma_{\mathrm{k}(\mathrm{n})}\right|^{\mathrm{uk}(\mathrm{n})} .
$$

So, if we take the sequence $\overline{\mathrm{w}}=\left(<\mathrm{x}_{\mathrm{k}}, \mathrm{y}_{\mathrm{k}}>\right)$ defined by

$$
\begin{aligned}
& <\mathrm{x}_{\mathrm{k}}, \mathrm{y}_{\mathrm{k}}>=\left\{\begin{array}{l}
-1 \\
\gamma_{\mathrm{k}(\mathrm{n})}<\mathrm{r}, \mathrm{t}>\text {, for } \mathrm{k}=\mathrm{k}(\mathrm{n}), \mathrm{n} \geq 1 \text {, and } \\
<0,0>\text {, otherwise. }
\end{array}\right. \\
& \text { where }<\mathrm{r}, \mathrm{t}>\in \mathrm{X} \times \mathrm{Y} \text { with }\|<\mathrm{r}, \mathrm{t}>\|=1 \text {, then we easily see that } \\
& \sup _{k}\left\|\gamma_{\mathrm{k}}<\mathrm{x}_{\mathrm{k}}, \mathrm{y}_{\mathrm{k}}>\right\|^{\mathrm{uk}}=\sup _{n}\left\|\gamma_{\mathrm{k}(\mathrm{n})}<\mathrm{x}_{\mathrm{k}(\mathrm{n})}, \mathrm{y}_{\mathrm{k}(\mathrm{n})}>\right\|^{\mathrm{uk}(\mathrm{n})} \\
& =\sup _{n}\|<\mathrm{r}, \mathrm{t}>\|^{\mathrm{uk}(\mathrm{n})} \quad=1
\end{aligned}
$$




$$
\begin{aligned}
& \text { and , } \quad \sup _{k}\left\|\mu_{\mathrm{k}}<\mathrm{x}_{\mathrm{k}}, \mathrm{y}_{\mathrm{k}}>\right\|^{\mathrm{uk}}=\sup _{n}\left\|\mu_{\mathrm{k}(\mathrm{n})}<\mathrm{x}_{\mathrm{k}(\mathrm{n})}, \mathrm{y}_{\mathrm{k}(\mathrm{n})}>\right\|^{\mathrm{uk}(\mathrm{n})} \\
& =\sup _{n}\left\{\left|\frac{\mu_{\mathrm{k}(\mathrm{n})}}{\gamma_{(\mathrm{n})}}\right| \mathrm{uk(n)}\|<\mathrm{r}, \mathrm{t}>\| \|^{\mathrm{uk}(\mathrm{n})}\right\} \\
& >\sup n=\infty \text {. }
\end{aligned}
$$

Hence $\overline{\mathrm{w}} \in \mathrm{V}(\mathrm{P}, \bar{\gamma}, \overline{\mathrm{u}},\|\cdot\|)$ but $\overline{\mathrm{w}} \notin \mathrm{V}(\mathrm{P}, \bar{\mu}, \overline{\mathrm{u}},\|\cdot\|)$, a contradiction. This completes the proof.

Theorem 3.4: For any $\overline{\mathrm{u}}=\left(\mathrm{u}_{\mathrm{k}}\right), \mathrm{V}(\mathrm{P}, \bar{\mu}, \overline{\mathrm{u}},\|\cdot\|) \subset \mathrm{V}(\mathrm{P}, \bar{\gamma}, \overline{\mathrm{u}},\|\cdot\|)$

if and only if $\lim _{k} \sup _{k}\left|\frac{\gamma_{\mathrm{k}}}{\mu_{\mathrm{k}}}\right|^{\mathrm{uk}}<\infty$.

\section{Proof :}

For the sufficiency, suppose lim $\sup _{k}\left|\frac{\gamma_{\mathrm{k}}}{\mu_{\mathrm{k}}}\right|^{\mathrm{uk}}<\infty$, and $\overline{\mathrm{w}}=\left(<\mathrm{x}_{\mathrm{k}}, \mathrm{y}_{\mathrm{k}}>\right) \in \mathrm{S}(\mathrm{X} \times \mathrm{Y}, \bar{\mu}, \overline{\mathrm{u}},\|\cdot\|)$.

Then there exists $\mathrm{L}>0$, such that

$\mathrm{L}\left|\mu_{\mathrm{k}}\right|^{\mathrm{uk}}>\left|\gamma_{\mathrm{k}}\right|^{\mathrm{uk}}$

for all sufficiently large values of $\mathrm{k}$. Thus

$$
\sup _{k}\left\|\gamma_{\mathrm{k}}<\mathrm{x}_{\mathrm{k}}, \mathrm{y}_{\mathrm{k}}>\right\|^{\mathrm{uk}} \leq \sup _{k} \mathrm{~L}\left\|\mu_{\mathrm{k}}<\mathrm{x}_{\mathrm{k}}, \mathrm{y}_{\mathrm{k}}>\right\|^{\mathrm{uk}}<\infty \text {, }
$$

for all sufficiently large values of $k$, implies that $\overline{\mathrm{w}} \in \mathrm{S}(\mathrm{X} \times \mathrm{Y}, \bar{\gamma}, \overline{\mathrm{u}},\|\cdot\|)$. Hence

$\mathrm{V}(\mathrm{P}, \bar{\mu}, \overline{\mathrm{u}},\|\cdot\|) \subset \mathrm{S}(\mathrm{P}, \bar{\gamma}, \overline{\mathrm{u}},\|\cdot\|)$.

For the necessity, suppose that

$\mathrm{V}(\mathrm{P}, \bar{\mu}, \overline{\mathrm{u}},\|\cdot\|) \subset \mathrm{S}(\mathrm{P}, \bar{\gamma}, \overline{\mathrm{u}},\|\cdot\|)$

but lim $\sup _{k}\left|\frac{\gamma_{k}}{\mu_{k}}\right|^{\text {uk }}=\infty$. Then we can find a sequence $(k(n))$ of positive integers

$\mathrm{k}(\mathrm{n})<\mathrm{k}(\mathrm{n}+1), \mathrm{n} \geq 1$

such that

$$
\mathrm{n}\left|\mu_{\mathrm{k}(\mathrm{n})}\right|^{\mathrm{uk}(\mathrm{n})}<\left|\gamma_{\mathrm{k}(\mathrm{n})}\right|^{\mathrm{uk}(\mathrm{n})} \text {, for each } \mathrm{n} \geq 1
$$

For $<\mathrm{r}, \mathrm{t}>\in \mathrm{X} \times \mathrm{Y}$ with $\|<\mathrm{r}, \mathrm{t}>\|=1$ we define sequence $\overline{\mathrm{w}}=\left(<\mathrm{x}_{\mathrm{k}}, \mathrm{y}_{\mathrm{k}}>\right)$ such that

$$
<\mathrm{x}_{\mathrm{k}}, \mathrm{y}_{\mathrm{k}}>=\left\{\begin{array}{c}
-1 \\
\mu_{\mathrm{k}(\mathrm{n})}<\mathrm{r}, \mathrm{t}>\text {, for } \mathrm{k}=\mathrm{k}(\mathrm{n}), \mathrm{n} \geq 1 \text {, and } \\
<0,0>\text {, otherwise. }
\end{array}\right.
$$

Then we easily see that

$$
\sup _{k}\left\|\mu_{\mathrm{k}}<\mathrm{x}_{\mathrm{k}}, \mathrm{y}_{\mathrm{k}}>\right\|^{\mathrm{uk}}=\sup _{n}\left\|\mu_{\mathrm{k}(\mathrm{n})}<\mathrm{x}_{\mathrm{k}(\mathrm{n})}, \mathrm{y}_{\mathrm{k}(\mathrm{n})}>\right\|^{\mathrm{uk}(\mathrm{n})}
$$




$$
=\sup _{n}\|<\mathrm{r}, \mathrm{t}>\|^{\mathrm{uk}(\mathrm{n})} \quad=1
$$

and

$$
\begin{aligned}
& \sup _{k}\left\|\gamma_{\mathrm{k}}<\mathrm{x}_{\mathrm{k}}, \mathrm{y}_{\mathrm{k}}>\right\|^{\mathrm{uk}}=\sup _{n}\left\|\gamma_{\mathrm{k}(\mathrm{n})}<\mathrm{x}_{\mathrm{k}(\mathrm{n})}, \mathrm{y}_{\mathrm{k}(\mathrm{n})}>\right\|^{\mathrm{uk}(\mathrm{n})} \\
& =\sup _{n}\left\{\left|\frac{\gamma_{\mathrm{k}(\mathrm{n})}}{\mu_{(\mathrm{n})}}\right| \mathrm{uk}(\mathrm{n})\|<\mathrm{r}, \mathrm{t}>\|^{\mathrm{uk}(\mathrm{n})}\right\} \\
& >\sup n=\infty \text {. }
\end{aligned}
$$

Hence $\overline{\mathrm{w}} \in \mathrm{V}(\mathrm{P}, \bar{\mu}, \overline{\mathrm{u}},\|\cdot\|)$ but $\overline{\mathrm{w}} \notin \mathrm{V}(\mathrm{P}, \bar{\gamma}, \overline{\mathrm{u}},\|\cdot\|)$, , which leads to a contradiction.

This completes the proof.

When Theorems 3.3 and 3.4 are combined, we get

Theorem 3.5: For any $\overline{\mathrm{u}}=\left(\mathrm{u}_{\mathrm{k}}\right), \mathrm{V}(\mathrm{P}, \bar{\gamma}, \overline{\mathrm{u}},\|\cdot\|)=\mathrm{V}(\mathrm{P}, \bar{\mu}, \overline{\mathrm{u}},\|\cdot\|)$

$$
\text { if and only if } 0<\lim \inf _{k}\left|\frac{\gamma_{\mathrm{k}}}{\mu_{\mathrm{k}}}\right|^{\mathrm{uk}} \leq \lim \sup _{k}\left|\frac{\gamma_{\mathrm{k}}}{\mu_{\mathrm{k}}}\right|^{\mathrm{uk}}<\infty \text {. }
$$

Corollary 3.6: For any $\overline{\mathrm{u}}=\left(\mathrm{u}_{\mathrm{k}}\right)$,

(i) $\quad \mathrm{V}(\mathrm{P}, \bar{\gamma}, \overline{\mathrm{u}},\|\cdot\|) \subset \mathrm{V}(\mathrm{P}, \overline{\mathrm{u}},\|\cdot\|)$ if and only if $\lim \inf _{k}\left|\gamma_{\mathrm{k}}\right|^{\mathrm{uk}}>0$;

(ii) $\mathrm{V}(\mathrm{P}, \overline{\mathrm{u}},\|\cdot\|) \subset \mathrm{V}(\mathrm{P}, \bar{\gamma}, \overline{\mathrm{u}},\|\cdot\|)$ if and only if $\lim \sup _{k}\left|\gamma_{\mathrm{k}}\right|^{\mathrm{uk}}<\infty$;

(iii) $\mathrm{V}(\mathrm{P}, \bar{\gamma}, \overline{\mathrm{u}},\|\cdot\|)=\mathrm{V}(\mathrm{P}, \overline{\mathrm{u}},\|\cdot\|)$ if and only if

$0<\lim \inf _{k}\left|\gamma_{\mathrm{k}}\right|^{\mathrm{uk}} \leq \lim \sup _{k}\left|\gamma_{\mathrm{k}}\right|^{\mathrm{uk}}<\infty$.

\section{Proof:}

Proof follows if we take $\mu_{\mathrm{k}}=1$ for all $\mathrm{k}$ in Theorems $3.3,3.4$ and 3.5 .

Theorem 3.7: For any $\bar{\gamma}=\left(\gamma_{\mathrm{k}}\right), \mathrm{V}(\mathrm{P}, \bar{\gamma}, \overline{\mathrm{u}},\|\cdot\|) \subset \mathrm{V}(\mathrm{P}, \bar{\gamma}, \overline{\mathrm{v}},\|\cdot\|)$

$$
\text { if and only if } \lim \sup _{k} \frac{\mathrm{v}_{\mathrm{k}}}{\mathrm{u}_{\mathrm{k}}}<\infty \text {. }
$$

\section{Proof:}

Let the condition hold. Then there exists $\mathrm{L}>0$ such that $\mathrm{v}_{\mathrm{k}}<\mathrm{Lu}_{\mathrm{k}}$ for all sufficiently large values of $\mathrm{k}$. Thus

$\sup _{k}\left\|\gamma_{k}<x_{k}, y_{k}>\right\|^{\text {uk }} \leq N$ for some $N>1$

implies that

$\sup _{k}\left\|\gamma_{\mathrm{k}}<\mathrm{x}_{\mathrm{k}}, \mathrm{y}_{\mathrm{k}}>\right\|^{\mathrm{vk}} \leq \mathrm{N}^{\mathrm{L}}$,

and hence $\quad \mathrm{V}(\mathrm{P}, \bar{\gamma}, \overline{\mathrm{u}},\|\cdot\|) \subset \mathrm{V}(\mathrm{P}, \bar{\gamma}, \overline{\mathrm{v}},\|\cdot\|)$. 
Conversely, let the inclusion hold but $\lim \sup _{k} \frac{\mathrm{v}_{\mathrm{k}}}{\mathrm{u}_{\mathrm{k}}}=\infty$. Then there exists a sequence $(\mathrm{k}(\mathrm{n}))$ of positive integers with

$$
\mathrm{k}(\mathrm{n})<\mathrm{k}(\mathrm{n}+1), \mathrm{n} \geq 1
$$

such that

$$
\mathrm{v}_{\mathrm{k}(\mathrm{n})}>\mathrm{n} \mathrm{u}_{\mathrm{k}(\mathrm{n})}, \mathrm{n} \geq 1 .
$$

We now define a sequence $\overline{\mathrm{w}}=\left(<\mathrm{x}_{\mathrm{k}}, \mathrm{y}_{\mathrm{k}}>\right)$ as follows:

$$
<x_{k}, y_{k}>=\left\{\begin{array}{l}
-1 \\
\gamma_{k}(n) 2^{1 / u k(n)}<r, t>, \text { for } k=k(n), n \geq 1 \\
<0,0>\text {, otherwise. }
\end{array}\right.
$$

where $<\mathrm{r}, \mathrm{t}>\in \mathrm{X} \times \mathrm{Y}$ with $\|<\mathrm{r}, \mathrm{t}>\|=1$.

Then for $k=k(n), n \geq 1$, we easily see that

$$
\begin{aligned}
& \sup _{k}\left\|\gamma_{\mathrm{k}}<\mathrm{x}_{\mathrm{k}}, \mathrm{y}_{\mathrm{k}}>\right\|^{\mathrm{uk}}=\sup _{n}\left\|\gamma_{\mathrm{k}(\mathrm{n})}<\mathrm{x}_{\mathrm{k}(\mathrm{n})}, \mathrm{y}_{\mathrm{k}(\mathrm{n})}>\right\|^{\mathrm{uk}(\mathrm{n})} \\
& =2 \sup _{n}\|<\mathrm{r}, \mathrm{t}>\|^{\mathrm{uk}(\mathrm{n})}=2 \\
& \sup _{k}\left\|\gamma_{\mathrm{k}}<\mathrm{x}_{\mathrm{k}}, \mathrm{y}_{\mathrm{k}}>\right\|^{\mathrm{vk}}=\sup _{n}\left\|\gamma_{\mathrm{k}(\mathrm{n})}<\mathrm{x}_{\mathrm{k}(\mathrm{n})}, \mathrm{y}_{\mathrm{k}(\mathrm{n})}>\right\|^{\mathrm{vk}(\mathrm{n})} \\
& =\sup _{n}\left\|2^{1 / \mathrm{uk}(\mathrm{n})}<\mathrm{r}, \mathrm{t}>\right\|^{\mathrm{vk}(\mathrm{n})} \\
& >\sup _{n} 2^{\mathrm{n}}=\infty \text {. }
\end{aligned}
$$

and ,

Hence $\overline{\mathrm{w}} \in \mathrm{V}(\mathrm{P}, \bar{\gamma}, \overline{\mathrm{u}},\|\cdot\|)$ but $\overline{\mathrm{w}} \notin \mathrm{V}(\mathrm{P}, \bar{\gamma}, \overline{\mathrm{v}},\|\|$.$) , a contradiction.$

This completes the proof.

Theorem 3.8: For any $\bar{\gamma}=\left(\gamma_{\mathrm{k}}\right), \mathrm{V}(\mathrm{P}, \bar{\gamma}, \overline{\mathrm{v}},\|\cdot\|) \subset \mathrm{V}(\mathrm{P}, \bar{\gamma}, \overline{\mathrm{u}},\|\cdot\|)$

$$
\text { if and only if } \lim \inf _{k} \frac{\mathrm{v}_{\mathrm{k}}}{\mathrm{u}_{\mathrm{k}}}>0 \text {. }
$$

\section{Proof:}

Let the condition hold and $\overline{\mathrm{w}}=\left(<\mathrm{x}_{\mathrm{k}}, \mathrm{y}_{\mathrm{k}}>\right) \in \mathrm{V}(\mathrm{P}, \bar{\gamma}, \overline{\mathrm{v}},\|\cdot\|)$. Then there exists $\mathrm{m}>0$ such that $\mathrm{v}_{\mathrm{k}}<$ $\mathrm{m} \mathrm{u}_{\mathrm{k}}$ for all sufficiently large values of $\mathrm{k}$ and

$\sup _{k}\left\|\gamma_{\mathrm{k}}<\mathrm{x}_{\mathrm{k}}, \mathrm{y}_{\mathrm{k}}>\right\|^{\mathrm{vk}} \leq \mathrm{N}$ for some $\mathrm{N}>1$.

This implies that

$$
\begin{aligned}
& \sup _{k}\left\|\gamma_{\mathrm{k}}<\mathrm{x}_{\mathrm{k}}, \mathrm{y}_{\mathrm{k}}>\right\|^{\mathrm{uk}} \leq \mathrm{N}^{1 / \mathrm{m}} \\
& \text { i.e, } \overline{\mathrm{w}}=\left(<\mathrm{x}_{\mathrm{k}}, \mathrm{y}_{\mathrm{k}}>\right) \notin \ell_{\infty}(\mathrm{X} \times \mathrm{Y}, \bar{\gamma}, \overline{\mathrm{u}}) \text { and hence } \\
& \mathrm{V}(\mathrm{P}, \bar{\gamma}, \overline{\mathrm{v}},\|\cdot\|) \subset \mathrm{V}(\mathrm{P}, \bar{\gamma}, \overline{\mathrm{u}},\|\cdot\|) .
\end{aligned}
$$


Conversely let the inclusion hold but $\lim \inf _{k} \frac{\mathrm{v}_{k}}{\mathrm{u}_{\mathrm{k}}}=0$. Then we can find a sequence $(\mathrm{k}(\mathrm{n}))$ of positive integers with $\mathrm{k}(\mathrm{n})<\mathrm{k}(\mathrm{n}+1), \mathrm{n} \geq 1$

such that

$\mathrm{n} \mathrm{v}_{\mathrm{k}(\mathrm{n})}<\mathrm{u}_{\mathrm{k}(\mathrm{n})}, \mathrm{n} \geq 1$.

Now taking $<\mathrm{r}, \mathrm{t}>\in \mathrm{P}$ with $\|<\mathrm{r}, \mathrm{t}>\|=1$, we define the sequence $\overline{\mathrm{w}}=\left(<\mathrm{x}_{\mathrm{k}}, \mathrm{y}_{\mathrm{k}}>\right)$ by

$$
<\mathrm{x}_{\mathrm{k}}, \mathrm{y}_{\mathrm{k}}>=\left\{\begin{array}{l}
-1 \\
\gamma_{\mathrm{k}(\mathrm{n})} 2^{1 / \mathrm{kk}(\mathrm{n})}<\mathrm{r}, \mathrm{t}>, \text { for } \mathrm{k}=\mathrm{k}(\mathrm{n}), \mathrm{n} \geq 1 \text {, and } \\
<0,0>\text {, otherwise. }
\end{array}\right.
$$

Then for $k=k(n), n \geq 1$, we easily see that

$$
\begin{aligned}
& \sup _{k}\left\|\gamma_{\mathrm{k}}<\mathrm{x}_{\mathrm{k}}, \mathrm{y}_{\mathrm{k}}>\right\|^{\mathrm{vk}}=\sup _{n}\left\|\gamma_{\mathrm{k}(\mathrm{n})}<\mathrm{x}_{\mathrm{k}(\mathrm{n})}, \mathrm{y}_{\mathrm{k}(\mathrm{n})}>\right\|^{\mathrm{vk}(\mathrm{n})} \\
& =2 \sup _{n}\|<\mathrm{r}, \mathrm{t}>\|^{\mathrm{vk}(\mathrm{n})}=2 \\
& \text { and } \\
& \sup _{k}\left\|\gamma_{\mathrm{k}}<\mathrm{x}_{\mathrm{k}}, \mathrm{y}_{\mathrm{k}}>\right\|^{\mathrm{uk}}=\sup _{n}\left\|\gamma_{\mathrm{k}(\mathrm{n})}<\mathrm{x}_{\mathrm{k}(\mathrm{n})}, \mathrm{y}_{\mathrm{k}(\mathrm{n})}>\right\|^{\mathrm{uk}(\mathrm{n})} \\
& =\sup _{n}\left\|2^{1 / \mathrm{vk}(\mathrm{n})}<\mathrm{r}, \mathrm{t}>\right\|^{\mathrm{uk}(\mathrm{n})} \\
& >\sup 2^{n}=\infty \text {. }
\end{aligned}
$$

Hence $\overline{\mathrm{w}} \in \mathrm{V}(\mathrm{P}, \bar{\gamma}, \overline{\mathrm{v}},\|\cdot\|)$ but $\overline{\mathrm{w}} \notin \mathrm{V}(\mathrm{P}, \bar{\gamma}, \overline{\mathrm{u}},\|\cdot\|)$, a contradiction.

This completes the proof.

On combining Theorems 3.7 and 3.8,we get the following theorem:

Theorem 3.9: For any $\bar{\gamma}=\left(\gamma_{\mathrm{k}}\right), \mathrm{V}(\mathrm{P}, \bar{\gamma}, \overline{\mathrm{u}},\|\cdot\|)=\mathrm{V}(\mathrm{P}, \bar{\gamma}, \overline{\mathrm{v}},\|\cdot\|)$

$$
\text { if and only if } 0<\lim \inf _{k} \frac{\mathrm{v}_{\mathrm{k}}}{\mathrm{u}_{\mathrm{k}}} \leq \lim \sup _{k} \frac{\mathrm{v}_{\mathrm{k}}}{\mathrm{u}_{\mathrm{k}}}<\infty \text {. }
$$

Corollary 3.10: For any $\bar{\gamma}=\left(\gamma_{\mathrm{k}}\right)$,

(i) $\mathrm{V}(\mathrm{P}, \bar{\gamma},\|\cdot\|) \subset \mathrm{V}(\mathrm{P}, \bar{\gamma}, \overline{\mathrm{u}},\|\cdot\|)$ if and only if $\lim \sup _{k} \mathrm{u}_{\mathrm{k}}<\infty$;

(ii) $\mathrm{V}(\mathrm{P}, \bar{\gamma}, \overline{\mathrm{u}},\|\cdot\|) \subset \mathrm{V}(\mathrm{P}, \bar{\gamma},\|\cdot\|)$ if and only if $\lim \inf _{k} \mathrm{u}_{\mathrm{k}}>0$;

(iii) $\mathrm{V}(\mathrm{P}, \bar{\gamma}, \overline{\mathrm{u}},\|\cdot\|)=\mathrm{V}(\mathrm{P}, \bar{\gamma},\|\cdot\|)$ if and only if

$0<\lim \inf _{k} \mathrm{u}_{\mathrm{k}} \leq \lim \sup _{k} \mathrm{v}_{\mathrm{k}}<\infty$.

\section{Proof:}

Proof easily follows when we take $\mathrm{u}_{\mathrm{k}}=1$ and $\mathrm{v}_{\mathrm{k}}=\mathrm{u}_{\mathrm{k}}$ for all $\mathrm{k}$ in theorem 3.7, 3.8 and 3.9. 
Theorem 3.11: For any sequences $\bar{\gamma}=\left(\gamma_{\mathrm{k}}\right), \bar{\mu}=\left(\mu_{\mathrm{k}}\right), \overline{\mathrm{u}}=\left(\mathrm{u}_{\mathrm{k}}\right)$ and $\overline{\mathrm{v}}=\left(\mathrm{v}_{\mathrm{k}}\right)$,

$\mathrm{V}(\mathrm{P}, \bar{\gamma}, \overline{\mathrm{u}},\|\cdot\|) \subset \mathrm{V}(\mathrm{P}, \bar{\mu}, \overline{\mathrm{v}},\|\cdot\|)$

if and only if (i) $\lim \inf _{k}\left|\frac{\gamma_{\mathrm{k}}}{\mu_{\mathrm{k}}}\right|^{\mathrm{uk}}>0$, and (ii) $\lim \sup _{k} \frac{\underline{\mathrm{v}}_{\mathrm{k}}}{\mathrm{u}_{\mathrm{k}}}<\infty$.

\section{Proof:}

Proof directly follows from Theorems 3.3 and 3.7.

\section{Conclusion}

In this paper, we have examined some conditions that characterize the linear space structures and containment relations of the space of sequences whose terms from a product normed space. In fact, these results can be used for further generalizations to investigate other properties of sequence spaces whose terms are in product normed spaces.

\section{References}

1. Castillo, J., Garcia, R. and Jaramillo, J.: "Extension of Bilinear Formson Banach Spaces”; Proceedings of the American Mathematical Society, 129(12), 3647- 3656, (2001)

2. Kamthan, P.K. and Gupta, M. "Sequence and series, lecture notes"; 65 Marcel Dekker Inc. Khan, V.A.(2008): "On a new sequence space defined by Orlicz functions”; Common Fac. Sci. Univ. Ank-series; 57( 2), 25-33, (2001)

3. Kolk,E. “Topologies in generalized Orlicz sequence spaces”; Filomat, 25(4), 191-211, (2011).

4. Köthe, G.: “Topological vector spaces”; Springer Verlag, Berlin Heidelberg, New York, (1970)

5. Maddox, I.J. "Infinite matrices of operators"; Lecture Notes in Mathematics 786, SpringerVerlag Berlin, Heidelberg, New York, ( 1980)

6. Malkowski, E. and Rakocevic, V." An introduction into the theory of sequence spaces and measures of non-compactness", (2004)

7 Ruckle. W.H. “Sequence spaces”, Pitman Advanced Publishing Programme, (1981)

8. Sanchez1, F., Garcia1, R.and Villanueva, I. "Extension of Multilinear Operators on Banach Spaces”; ExtractaMathematica; 15, (2), 291 - 334, (2000)

9 Srivastava,J.K. and Pahari,N.P.: "On vector valued paranormed sequence space $c_{0}(X, M, \bar{\lambda}, \bar{p}$ ) defined by Orlicz function”; J.Rajasthan Acad. of Phy. Sci.;11(2),11-24, (2012)

10. Wilansky, A. "Modern methods in topological vector spaces”; McGraw-Hill Book Co. Inc. New York, (1978)

11. Yilmaz, Y. and Solak,I. "Operator Perfectness and Normality of Vector-Valued Sequence Spaces”; Thai Journal of Mathematics; 2 (2) : 247-257, (2004) 